\title{
Primary intent vaginal hysterectomy: outcomes for common contraindications to vaginal approach hysterectomy
}

\author{
Malcolm W. Mackenzie • Jeffrey D. Johnson
}

Received: 18 July 2010 / Accepted: 16 August 2010 / Published online: 7 September 2010

(C) Springer-Verlag 2010

\begin{abstract}
The objective of this study is, within a broadly inclusive selection strategy for benign vaginal hysterectomy, to determine whether the most commonly invoked "contraindications" to vaginal hysterectomy-fibroid enlargement $>14$ weeks, prior cesarean, need for oophorectomy-result in increased risk of complications. This study is of retrospective design within a rural community hospital. All vaginal hysterectomies performed by a single practitioner over an 11-year-period (1998-2009) were used as samples in this study. With few exclusions, all candidates for benign hysterectomy underwent vaginal hysterectomy. Comparison was made between vaginal cases without enlargement $>14$ weeks, prior cesarean, or need for oopherectomy defined as "Standard" and those with contraindications defined as "Non-standard." Intraoperative complications and morbidity, including conversion to abdominal route, and postoperative morbidity, including return to the OR, transfusions, and length of hospital stay, were the main outcome measures. Of 325 hysterectomies attempted vaginally during the study period, 165 were classified as "Standard" and 160 classified as "Non-
\end{abstract}

\author{
M. W. Mackenzie $(\bowtie) \cdot J$. D. Johnson \\ Department of Obstetrics and Gynecology, \\ Cheshire Medical Center/Dartmouth Hitchcock, \\ Keene, NH, USA \\ e-mail: mmacken1@mah.harvard.edu \\ M. W. Mackenzie · J. D. Johnson \\ Department of Obstetrics and Gynecology, \\ Mount Auburn Hospital, \\ 330 Mount Auburn St, \\ Cambridge, MA, USA \\ Present Address: \\ M. W. Mackenzie \\ 836 Nelson Rd, \\ Nelson, NH 03457, USA
}

standard." Hysterectomy was completed vaginally in 311 $(95.7 \%)$ patients, while $14(4.3 \%)$ required abdominal conversion; more common for the non-standard group $(8.1 \%$ vs. $0.6 \%, p<0.05)$. Complications not requiring conversion were not different. Only operative time, EBL, and uterine weight were increased for the non-standard group $(p<0.05)$. No differences were seen in length of stay, early, or late postoperative complications. Uterine enlargement $>14$ weeks, prior cesarean, or oophorectomy conventionally contraindicates vaginal hysterectomy; a primary intent vaginal hysterectomy strategy using broad inclusion criteria results in a high vaginal hysterectomy rate, and low complication rates no greater for vaginal hysterectomies performed with contraindications than for those performed without such contraindications.

Keywords Vaginal hysterectomy - Contraindications . Fibroid · Cesarean · Oophorectomy

\section{Introduction}

In the USA, over 600,000 hysterectomies are performed annually, making it second only to cesarean delivery among surgeries performed on women [1]. The abdominal approach, the most commonly performed [2], carries the highest complication rate [3] and imposes the greatest recovery burden and highest cost [4]. Laparoscopic hysterectomy, potentially a less invasive alternative to abdominal hysterectomy, has suffered from slow skill development [5] and higher complication rates [6] and cost as compared to the vaginal approach [7]. Vaginal hysterectomy, with its lowest rate of complications, recovery burden, and costs $[8,9]$, might be considered the optimal approach. Mindful, the American College of Obstetricians and Gynecologists, 
in its November 2009 Committee Opinion \#444 regarding hysterectomy route for benign indications states, "evidence demonstrates that, in general, vaginal hysterectomy is associated with better outcomes and fewer complications than laparoscopic or abdominal hysterectomy" concluding "vaginal hysterectomy is the approach of choice whenever feasible, based on its well-documented advantages and lower complication rates" [10].

Many are the claimed "contraindications" to a vaginal approach hysterectomy; any complexity beyond the normal sized, unscarred, descended uterus, without indication for oophorectomy dissuades many practitioners from attempting vaginal hysterectomy. The clinical reality is that conditions which constitute for many surgeons "contraindications" to vaginal hysterectomy-fibroid enlargement, prior cesarean delivery or pelvic surgery, and need for oophorectomy - are commonly present in the hysterectomy candidate. In consequence, rates of vaginal hysterectomy, despite being an optimal approach by all criteria, are lowno greater than 20-25\% - a fact bemoaned by those promoting a best practice standard of $75-80 \%$ vaginal hysterectomy rate $[11,12]$.

We report the outcomes of a hysterectomy strategy that preferentially and primarily pursues a vaginal approach where neither fibroid enlargement to 18 -week size, prior history of cesarean delivery, or need for oophorectomy (much less prior pelvic surgery, known adhesions, endometriosis, nulliparity, obesity, or absence of uterine descent) are considered dissuasive from initiating vaginal hysterectomy and to determine whether any greater risk of complications accrues to vaginal hysterectomies challenged by such "contraindications" than to vaginal hysterectomies not so challenged.

\section{Methods}

Following approval from Dartmouth Hitchcock Medical Center's Committee for Protection of Human Subjects, all hysterectomies performed by one gynecologic surgeon at The Cheshire Medical Center/Dartmouth Hitchcock-Keene were identified for the dates February 1, 1998 to February 28, 2009.

Patients undergoing abdominal approach hysterectomies (open and laparoscopic) were identified and their charts reviewed to determine the indication for the abdominal approach. For the remaining necessarily vaginal hysterectomies-performed, following standard bowel prep and protocol-driven antibiotic prophylaxis, with standard technique notable for intrafascial dissection and delayed peritoneal entry - inpatient and outpatient records were reviewed. All measures bearing on the feasibility of and those bearing on the morbidity potentially consequent upon a vaginal approach were extracted: preoperative data including patient age, body mass index (BMI), gravida, para, number of vaginal deliveries, number of cesarean deliveries, preoperatively estimated uterine size (in weeks), hemoglobin and hematocrit, presence and degree of uterine descent (traditional grading system grade $0-4$ ), presence and degree of coincidental pelvic floor abnormalities (cystocele/rectocele), prior pelvic surgeries with or without known intrapelvic adhesive disease, and preoperatively identified indication(s) for hysterectomy: fibroid-related symptoms (bleeding, mass effect, pain), non-fibroid bleeding, non-fibroid pain, non-fibroid dysmenorrhea, known endometriosis, known adenomyosis, and pelvic floor dysfunction/prolapse. Intraoperative events were recorded: estimated blood loss (EBL), total operative time (OR) from first cut to finish of surgery, concomitant procedures (oophorectomy, pelvic floor repairs including anterior and posterior repairs and sacrospinous ligament suspension), method of anesthesia, intraoperative complications, need for conversion to abdominal route, and uterine weight. Postoperative events were identified: immediate (during hospitalization), early (discharge to 3 months postoperative) and late (3 to 12 months postoperative) complications including returns to the OR, transfusions, ICU admissions, and hospital readmissions. Also recorded were length of hospital stay (LOS), years of follow-up, and the occurrence/ recurrence of symptomatic prolapse.

Averages with standard deviations were calculated for continuous variable data while rates and percentages were calculated for categorical or binary data.

Based upon preoperative criteria, and focusing on commonly invoked contraindications to vaginal route hysterectomy, patients were then divided into two groups:

Standard: vaginal hysterectomies without "contraindication" (size less than 15 weeks, no history of cesarean, no oophorectomy)

Non-standard: vaginal hysterectomies performed despite "contraindications" (size 15 weeks or greater, and/or prior cesarean, and/or oophorectomy)

Utilizing Excel (Microsoft Corp, USA), comparison between these "Standard" and "Non-standard" hysterectomy groups was conducted in terms of preoperative, intraoperative, and postoperative criteria. Comparative rates with significance for non-continuous data were calculated using Yates' chi-squared analysis. Significance testing utilizing two-tailed Student's $t$ test was applied to the comparison of continuous data.

An additional analysis, intended to identify the morbidity consequent upon intraoperative conversion to abdominal route, compares "Non-standard" hysterectomies that resulted in a conversion to abdominal route against the group of "Non-standard" hysterectomies that were completed vaginally. 


\section{Results}

There were 405 hysterectomies performed during the study period. For 80 hysterectomies, no vaginal approach was attempted: 17 for known or suspected malignancy, 13 for uterine size $\geq 19$ weeks, seven for uterine size $<19$ weeks but with fibroid distortion of the ureter/uterine artery nexus, nine for laparoscopic supracervical hysterectomy (no total laparoscopic hysterectomies nor laparoscopically assisted vaginal hysterectomies were performed), 18 where planned urogynecologic procedure directed an abdominal approach, and three for history of severe sexual abuse out of concern for consequent posttraumatic stress. Amongst general surgical indications, abdominal hernia repair, small bowel obstruction, and pelvic abscess directed an abdominal approach in 13 other cases.

For 325 patients, primary intent vaginal approach hysterectomy was attempted. Demographic and outcome data for this primary intent vaginal hysterectomy group is seen in Table 1 .

\section{Preoperative data}

Of these 325 primary intent vaginal hysterectomies, 80 (24.6\%) had a history of C/S (27 (8.3\%) with one cesarean delivery, 37 (11.4\%) with two cesareans, $11(3.4 \%)$ with three, and five (1.5\%) with four). Eighty-nine (27.4\%) patients registered body mass indices in the range of $30-39.9 \mathrm{~kg} / \mathrm{m}$ [2] (obesity) and $37(11.4 \%)$ patients evidenced a BMI in excess of $40 \mathrm{~kg} / \mathrm{m}$ [2] (morbid obesity). Twenty-five (7.7\%) patients were nulligravid. One hundred (30.1\%) patients had preoperatively identified uterine enlargement: $60(18.5 \%)$ had enlargement that was assessed as $8-12$ weeks and 40 $(12.3 \%)$ assessed at a 13-18-week size, of which 17 $(5.2 \%)$ were 15 weeks or greater. One hundred fifty-seven (48.3\%) patients had a prior history of some type of pelvic surgery of which 48 (14.8\%) were major surgeries. Pelvic adhesions were known to be present in 33 (10.1\%) patients. Amongst the standard indications for hysterectomy, the most common was non-fibroid bleeding (29.8\%).

\section{Intraoperative data}

There were 14 (4.3\%) conversions to abdominal approachutilizing laparoscopy (2) or laparotomy (12) - for reasons including intraoperative discovery of occult malignancy (2), bleeding from adnexa (2), technical inability to complete hysterectomy(5), technical inability to complete adnexectomy (4), and cystotomy (1) unable to be repaired vaginally. During the period of study, one additional cystotomy and no ureteral injuries occurred for a total urologic injury rate of $0.6 \%$. Average blood loss for the total group of patients was $140 \mathrm{~cm}^{3}$. Average operating time was 105 min (range 39$332 \mathrm{~min}$ ); this included in all patients hysterectomy, cul-de- sac obliteration, McCall, and round ligament resuspension, in addition to concomitant adnexectomy and pelvic reconstructive procedures. Ninety $(27.7 \%)$ hysterectomies also involved some manner of unilateral or bilateral oophorectomy. Obviating the performance of McCall culdoplasty, sacrospinous ligament suspension was performed in 21 $(6.5 \%)$ cases. Spinal anesthesia was utilized in $53(16.3 \%)$ patients with conversion to general anesthesia necessary in ten $(3.1 \%)$ patients.

\section{Postoperative data}

Immediate postoperative complications involved two (0.6\%) patients returning to the OR for laparoscopy, both for infundibulopelvic bleeding. The only transfusions were for these same two patients. No ICU admissions were necessary in any of the 325 patients. Median length of hospital stay for all patients was 1 day, range $0-7$ days. The total postdischarge (early and late) complication rate was 2.5\% involving eight patients. Early postoperative complications included one readmission for pyelonephritis on day 5 postoperative, one readmit at 2 weeks postoperative for vaginal apex bleeding requiring oversewing of the cuff. One patient required readmission for pain control 3 days following surgery and this same patient was readmitted on day 10 for Clostridium difficile colitis. C. difficile colitis necessitated admission for one other patient at day 10 postoperative. Pelvic abscess requiring laparotomy and drainage was seen in one patient 2 weeks postoperative. Late postoperative complications included one fatal myocardial infarction in a 72-year-old 10 months postoperative and one death from non-gynecologic carcinoma. One patient with ovarian remnant required laparoscopic excision at 8 months following hysterectomy. Mean uterine weight for all patients was $157 \mathrm{~g}$ (range 23-913 g). An average of 4.3 years of followup was achieved in 304 patients with 15 (5.0\%) patients evidencing symptomatic recurrent or new prolapse.

"Standard" vs. "Non-standard"

From this group of 325 vaginal hysterectomy patients, 160 were designated as non-standard cases - based upon the presence of at least one of the contraindications of prior cesarean, uterine enlargement greater than 14 weeks, and planned adnexectomy - with the remaining 165 patients without such contraindication designated standard. Comparison between these groups and statistical analysis is presented in Table 2.

In terms of preoperative criteria, significant differences in patient age, uterine descent or other pelvic floor defects, pelvic adhesions, fibroid uterus, and endometriosis were evident. Endometriosis was a very common indication for hysterectomy (necessarily with BSO), significantly more 
Table 1 All vaginal hysterectomies $(n=325)$

\begin{tabular}{|c|c|c|c|c|}
\hline & Avg. & SD & Present & $\%$ \\
\hline \multicolumn{5}{|l|}{ Preoperative: } \\
\hline Age (years) & 44.9 & 11.9 & & \\
\hline BMI $\left(\mathrm{kg} / \mathrm{m}^{2}\right)$ & 29.6 & 7.9 & & \\
\hline Gravida \# & 2.5 & 1.4 & & \\
\hline Para. \# & 2.2 & 1.2 & & \\
\hline Vag delivery: \# of pts. with any Vag. Del. & & & 239 & 73.5 \\
\hline Cesarean delivery: \# of pts. with any $\mathrm{C} / \mathrm{S}$ & & & 80 & 24.6 \\
\hline $\operatorname{Hgb}(\mathrm{mg} / \mathrm{dl})$ & 13.2 & 1.4 & & \\
\hline Het $(\%)$ & 40 & 21.1 & & \\
\hline Any uterine descent & & & 131 & 40.3 \\
\hline Other pelvic floor defects & & & 44 & 13.5 \\
\hline Prior major pelvic surgeries & & & 48 & 14.8 \\
\hline Prior minor pelvic surgeries (1) & & & 109 & 33.5 \\
\hline Pelvic adhesions known present & & & 33 & 10.1 \\
\hline Pelvic adhesions known absent (2) & & & 23 & 7.1 \\
\hline Fibroids & & & 79 & 24.3 \\
\hline Non-fibroid bleeding & & & 97 & 29.8 \\
\hline Non-fibroid pain & & & 20 & 6.2 \\
\hline Non-fibroid dysmenorrhea & & & 49 & 15.1 \\
\hline Endometriosis & & & 47 & 14.5 \\
\hline Adenomyosis & & & 50 & 15.4 \\
\hline Prolapse & & & 67 & 20.6 \\
\hline Other & & & 24 & 7.4 \\
\hline \multicolumn{5}{|l|}{ Intraoperative: } \\
\hline Conversion to abdominal route \# & & & 14 & 4.3 \\
\hline $\operatorname{EBL}\left(\mathrm{cm}^{3}\right)$ & 140 & 152 & & \\
\hline Complications w/out conversion & & & 1 & 0.3 \\
\hline OR time $(\mathrm{min})$ & 105 & 45 & & \\
\hline LSO or RSO & & & 30 & 9.2 \\
\hline $\mathrm{BSO}$ & & & 60 & 18.5 \\
\hline Anterior repair & & & 1 & 0.3 \\
\hline Posterior repair & & & 23 & 7.1 \\
\hline Anterior and posterior repair & & & 9 & 2.8 \\
\hline Sacrospinous ligament suspension & & & 21 & 6.5 \\
\hline Any pelvic floor repair & & & 37 & 11.4 \\
\hline \multicolumn{5}{|l|}{ Anesthesia: } \\
\hline GET & & & 230 & 70.8 \\
\hline GLMA & & & 52 & 16 \\
\hline Spinal & & & 53 & 16.3 \\
\hline Uterine weight (g) & 157 & 140 & & \\
\hline \multicolumn{5}{|l|}{ Postoperative: } \\
\hline Return to OR & & & 2 & 0.6 \\
\hline Transfusion & & & 2 & 0.6 \\
\hline ICU admit & & & 0 & 0 \\
\hline Hospital LOS (days) & 1.5 & 0.7 & & \\
\hline Early and late postoperative complications & & & 8 & 2.5 \\
\hline Follow-up: $>1$ year $(3)$ & & & 258 & 79.4 \\
\hline Length of long-term follow-up (years) & 4.3 & 2.6 & & \\
\hline Apical descent in pts. with any follow-up & & & 15 & 4.9 \\
\hline
\end{tabular}


Table 1 (continued)

Avg.

SD

Present

$\%$

(1) No prior surgeries: $168(51.7 \%)$

(2) Adhesions unknown: 269 (82.8\%)

(3) Follow-up: $<1$ year=46 (14.2\%); None=21 (6.5\%)

common $(26.9 \%)$ in the non-standard group than in the standard group (2.4\%).

The rate of conversion to abdominal route was higher, operative time longer - though with comparable ranges of 47-331 min vs. 39-332min - and average uterine weight greater in the non-standard as compared to the standard group. The use of spinal anesthesia and pelvic reconstructive procedures were more common in the standard group. Excluding intraoperatively discovered occult malignancy as the indication for conversion $(n=2)$, the rate of conversion for the non-standard group recalculates to a rate of $6.7 \%$ still significantly $(p<0.05)$ higher than in the standard group. Aside from conversion to abdominal route, no intraoperative complications were encountered in the standard group while a cystotomy repaired vaginally occurred in the non-standard group; the total intraoperative complication rate - both those necessitating conversion and those not-were significantly lower in the standard group $(0.6 \%)$ than in the non-standard group $(8.7 \%)$. Though both urologic injuries occurred in the non-standard group, the calculated rates of urologic injury were not significantly different $(p=0.47)$. There were significant differences between the two groups in terms of average operative time: for the standard and for the non-standard vaginal hysterectomies, 96 and $114 \mathrm{~min}$, respectively. Significantly more pelvic reconstructive procedures were performed in the standard group (19.4\%) than the non-standard group (3.1\%).

The 1.5-day average length of hospital stay was identical between groups. An equal number and rate $(0.6 \%)$ of immediate postoperative complications (requiring transfusion and return to the OR) occurred in each group. Neither was there significant difference in the combined (immediate, early, and late) postoperative complication rate between the standard $(1.2 \%)$ and non-standard (5\%) groups. The single early complication in the standard group involved bleeding from the vaginal apex (requiring oversewing of the cuff) encountered 2 weeks postoperative. All remaining and previously identified complications occurred in the non-standard group. Long-term follow-up beyond 1 year was known for 153 (92.7\%) of the standard group and 105 (66\%) of the non-standard group $(p<0.05)$ with the average interval to follow-up similar. For those with known follow-up beyond 1 year, recurrent or new symptomatic vault descent occurred significantly more often (compare $8.5 \%$ to $1.9 \%$ ) in the standard than in the non-standard group.

Finally, to determine whether certain risk factors for conversion to abdominal route can be identified from preoperative conditions and whether there are significant differences in postoperative outcomes, non-standard hysterectomies were separated according to whether conversion to abdominal route was necessary or not; this data appears in Table 3. As indications for hysterectomy, nonfibroid pelvic pain and an absence of uterine descent and were significantly more common in the group for whom conversion was necessary. Intraoperative complications (bleeding or bladder injury) were significantly more common in the conversion group $(23.1 \%)$ as compared to the non-conversion group $(0.6 \%)$. Not surprisingly, OR time, EBL, and LOS were all significantly greater for cases involving conversion than for cases successfully completed vaginally.

\section{Discussion}

This study reports the outcomes of one practitioner's strategy of preferentially pursuing vaginal approach for benign hysterectomy; a selection strategy driven by inclusive primary intent rather than exclusionary contraindications. By attempting a vaginal approach to hysterectomy despite commonly invoked contraindications - uterine enlargement $>14$ weeks, concomitant oophorectomy, or prior cesarean delivery-one of the highest published vaginal hysterectomy rates was achieved. We demonstrate that vaginal hysterectomy cases challenged by contraindications evidence slightly higher rates of conversion to abdominal approach than those cases not so challenged; we also identify no other significant differences in morbidity of clinical import. In conclusion, the three most commonly invoked contraindications to vaginal approach hysterectomy need not be dissuasive to primarily attempting a vaginal approach.

Many authors identify that, utilizing certain techniques, completion of vaginal hysterectomy is possible even for fibroid enlarged uteri over a range of sizes, with cutoffs set at 12 [11], 14 [13], 16 [12], or even 20 [14]-week size. Some authors also attest to the ease of transvaginal 
Table 2 Vaginal hysterectomies: "Non-Standard" vs. "Standard"

\begin{tabular}{|c|c|c|c|c|c|c|c|c|c|}
\hline & \multirow[t]{2}{*}{$p$} & \multicolumn{4}{|c|}{ Non-standard $(n=160)$} & \multicolumn{4}{|c|}{ Standard $(n=165)$} \\
\hline & & Avg & SD & Present & $\%$ & Avg & SD & Present & $\%$ \\
\hline \multicolumn{10}{|l|}{ Preoperative: } \\
\hline Age (years) & $<0.01$ & 42.8 & 11 & & & 46.9 & 12.4 & & \\
\hline BMI $\left(\mathrm{kg} / \mathrm{m}^{2}\right)$ & NS & 30.4 & 7.9 & & & 28.8 & 7.8 & & \\
\hline Gravida \# & NS & 2.5 & 1.3 & & & 2.6 & 1.5 & & \\
\hline Para \# & NS & 2.2 & 1.1 & & & 2.3 & 1.2 & & \\
\hline $\mathrm{Hgb}(\mathrm{mg} / \mathrm{dl})$ & NS & 13.1 & 1.4 & & & 13.3 & 1.4 & & \\
\hline Hct $(\%)$ & NS & 38.8 & 3.6 & & & 41.3 & 29.4 & & \\
\hline Any uterine descent & $<0.001$ & & & 43 & 26.9 & & & 88 & 53.3 \\
\hline Other pelvic floor defects & $<0.001$ & & & 6 & 3.8 & & & 38 & 23 \\
\hline Prior pelvic surgeries: major & NS & & & 23 & 14.4 & & & 25 & 15.2 \\
\hline Prior pelvic surgeries: minor & NS & & & 52 & 32.5 & & & 57 & 34.5 \\
\hline Intrapelvic adhesions known present & $<0.05$ & & & 24 & 15 & & & 9 & 5.4 \\
\hline Intrapelvic adhesions known absent & & & & 12 & 7.5 & & & 11 & 6.7 \\
\hline Fibroids & $<0.05$ & & & 48 & 30 & & & 31 & 18.8 \\
\hline Non-fibroid bleeding & NS & & & 45 & 28.1 & & & 52 & 31.5 \\
\hline Non-fibroid pain & NS & & & 13 & 8.1 & & & 7 & 4.2 \\
\hline Non-fibroid dysmenorrhea & NS & & & 26 & 16.2 & & & 23 & 13.9 \\
\hline Endometriosis & $<0.001$ & & & 43 & 26.9 & & & 4 & 2.4 \\
\hline Adenomyosis & NS & & & 26 & 16.3 & & & 24 & 14.5 \\
\hline Pelvic floor dysfunction or prolapse & $<0.001$ & & & 11 & 6.9 & & & 56 & 33.9 \\
\hline Other & NS & & & 9 & 5.6 & & & 15 & 9.1 \\
\hline \multicolumn{10}{|l|}{ Intraoperative: } \\
\hline Conversion to abdominal route & $<0.05$ & & & 13 & 8.1 & & & 1 & 0.6 \\
\hline $\operatorname{EBL}\left(\mathrm{cm}^{3}\right)$ & $<0.05$ & 166 & 172 & & & 115 & 125 & & \\
\hline Complications w/out conversion & NS & & & 1 & 0.6 & & & 0 & 0 \\
\hline OR time $(\mathrm{min})$ & $<0.001$ & 114 & 47 & & & 96 & 42 & & \\
\hline Any pelvic floor repair & $<0.001$ & & & & 5 & 3.1 & & 32 & 19.4 \\
\hline Uterine weight $(\mathrm{g})$ & $<0.001$ & 185 & 167 & & & 130 & 101 & & \\
\hline \multicolumn{10}{|l|}{ Postoperative: } \\
\hline Transfusion & NS & & 1 & 0.6 & & 1 & 0.6 & & \\
\hline Hospital LOS (days) & NS & 1.5 & 0.7 & & & 1.5 & 0.8 & & \\
\hline Return to OR & NS & & 1 & 0.6 & & & & 1 & 0.6 \\
\hline Early and late postoperative complications & NS & & 7 & 4.4 & & & & 1 & 0.6 \\
\hline Length of long-term follow-up (years) & NS & 4.3 & 2.6 & & & 4.3 & 2.6 & & \\
\hline Descent in pts. with $>1$ year follow-up & $<0.05$ & & & 2 & 1.9 & & & 13 & 8.5 \\
\hline
\end{tabular}

oopherectomy $[15,16]$ and some identify a history of cesarean delivery and the consequent scarring of cystouterine dissection planes as being of no impediment to vaginal hysterectomy [17]. They argue that singly each of these contraindications should not dissuade otherwise skilled surgeons from vaginal approach. The clinical reality however is that multiple contraindications are often present in candidates for hysterectomy. The data presented here argues for a selection strategy for vaginal approach most broadly applicable even when overlapping contraindications apply.
This study extends the work of previous authors who also challenge commonly invoked contraindications to vaginal approach: enlarged uteri $>180 \mathrm{~g}$, functional or actual nulliparity, and previous cesarean or pelvic laparotomy [18]. In our study, however, we chose the most commonly invoked contraindications to vaginal routeoophorectomy, prior cesarean delivery, and uterine enlargement $>14$ weeks. We did not address less commonly invoked contraindications to vaginal hysterectomy - nulliparity, obesity, absence of uterine descent or vaginal access, history of 
Table 3 Nonstandard hysterectomies: no abdominal conversion vs. conversion

\begin{tabular}{|c|c|c|c|c|c|c|c|c|c|}
\hline & \multirow[t]{2}{*}{$p$} & \multicolumn{4}{|c|}{ No conversion $(n=146)$} & \multicolumn{4}{|c|}{ Conversion $(n=14)$} \\
\hline & & Avg & SD & Present & $\%$ & Avg & $\mathrm{SD}$ & Present & $\%$ \\
\hline \multicolumn{10}{|l|}{ Preoperative: } \\
\hline Age (years) & NS & 42.4 & 10.7 & & & 47.2 & 13.1 & & \\
\hline BMI $\left(\mathrm{kg} / \mathrm{m}^{2}\right)$ & NS & 30.2 & 7.9 & & & 32.8 & 8.4 & & \\
\hline Grav. \# & NS & 2.5 & 1.3 & & & 2.5 & 1.3 & & \\
\hline Para. \# & NS & 2.2 & 1.1 & & & 2.2 & 1 & & \\
\hline Vag delivery \# per & NS & 1.2 & 1.3 & & & 1.3 & 1.3 & & \\
\hline Cesarean delivery \# per & NS & 1 & 1.1 & & & 0.8 & 1.3 & & \\
\hline History of any cesarean & NS & & & 75 & 46.4 & & & 5 & 38.4 \\
\hline $\operatorname{Hgb}(\mathrm{mg} / \mathrm{dl})$ & NS & 13.1 & 1.4 & & & 13.5 & 1.2 & & \\
\hline Het $(\%)$ & NS & 38.6 & 3.7 & & & 40.3 & 2.9 & & \\
\hline Any uterine descent & $<0.001$ & & & 39 & 26.5 & & & 4 & 30.8 \\
\hline Other pelvic floor defects & NS & & & 5 & 3.4 & & & 1 & 7.7 \\
\hline Prior pelvic surgeries: major & NS & & & 23 & 15.6 & & & 0 & 0 \\
\hline Prior pelvic surgeries:minor & NS & & & 50 & 32.7 & & & 4 & 30.8 \\
\hline Intrapelvic adhesions known present & NS & & & 22 & 15 & & & 2 & 15.3 \\
\hline Intrapelvic adhesions known absent & NS & & & 10 & 6.8 & & & 2 & 15.4 \\
\hline Fibroids & NS & & & 42 & 28.6 & & & 6 & 46.2 \\
\hline Non-fibroid bleeding & NS & & & 42 & 28.6 & & & 3 & 23.1 \\
\hline Non-fibroid pain & $<0.01$ & & & 9 & 6.1 & & & 4 & 30.8 \\
\hline Non-fibroid dysmenorrhea & NS & & & 26 & 17.7 & & & 0 & 0 \\
\hline Endometriosis & NS & & & 40 & 27.2 & & & 3 & 23.1 \\
\hline Adenomyosis & NS & & & 25 & 17 & & & 1 & 7.7 \\
\hline Pelvic floor dysfunction or prolapse & NS & & & 11 & 7.5 & & & 0 & 0 \\
\hline Other & NS & & & 8 & 5.4 & & & 1 & 7.7 \\
\hline \multicolumn{10}{|l|}{ Intraoperative: } \\
\hline $\mathrm{EBL}(\mathrm{mg} / \mathrm{dl})$ & $<0.01$ & 141 & 119 & & & 448 & 357 & & \\
\hline Complications w/out conversion & $<0.05$ & & & 1 & 0.6 & & & 3 & 23.1 \\
\hline OR time $(\mathrm{min})$ & $<0.001$ & 107 & 40.4 & & & 191 & 47 & & \\
\hline Any adnexectomy & NS & & & 79 & 53.7 & & & 11 & 84.6 \\
\hline Any pelvic floor repair & NS & & & 4 & 2.7 & & & 1 & 7.7 \\
\hline Uterine weight (gms) & NS & 177 & 155 & & & 278 & 254 & & \\
\hline$\#$ pts. with uterine weight $>200 \mathrm{~g}$ & NS & & & 113 & 76.9 & & & 8 & 62 \\
\hline \multicolumn{10}{|l|}{ Postoperative: } \\
\hline Transfusion & NS & & & 1 & 0.6 & & & 0 & 0 \\
\hline ICU admit & & & & 0 & 0 & & & 0 & 0 \\
\hline Hospital LOS (days) & $<0.05$ & 1.41 & 0.62 & & & 2.2 & 0.9 & & \\
\hline Return to OR & NS & & & 1 & 0.6 & & & 0 & 0 \\
\hline Late postoperative complications & NS & & & 5 & 3.4 & & & 2 & 15.4 \\
\hline
\end{tabular}

prior major pelvic surgery, and known or unknown adhesive disease. Though such lesser contraindications were not differentially represented between the two comparative groups, neither were they dissuasive against a primary intent vaginal approach.

A uterine size cutoff of greater than 18 weeks was chosen as the limit of this practitioner's intent to perform hysterec- tomy vaginally. The 18 -week cutoff however extends most previously published cutoffs as proof of the validity of more liberal criteria for inclusion vs. exclusion from vaginal approach.

Suggested here, the broadest applicability of a vaginal approach to hysterectomy challenges practitioners to examine what skills are required for a primary intent vaginal 
approach to hysterectomy. This has been answered by recent authors who point out that "attitude" [12] or "physician-level factors" [19] have perhaps greater influence on the choice of hysterectomy route than does actual "aptitude"; gynecologic surgeons have the requisite skill set and can, with a change of "attitude" or focus on evidencebased approaches [20, 21], increase substantially their vaginal hysterectomy rate. Additionally, by this study we extend the challenge to include an even larger group of patients for whom vaginal hysterectomy is entirely appropriate. That this group of patients with all ranges of uterine size, need for oophorectomy, and history of cesarean delivery - not to mention conditions of nulliparity, obesity, absence of descent, history of prior major pelvic surgery with known or unknown adhesive disease, etc. that we did not focus on at all-was treated successfully with vaginal hysterectomy implies then the concept of a "trial of vaginal hysterectomy" rate as a measure of quality different than the rate of "vaginal hysterectomy": for this study the former "trial" rate is calculated to be $80.2 \%$ and the latter rate of "completion" is calculated as a "vaginal hysterectomy" rate of $95.7 \%$.

This study evaluated the broadest range of relevant outcomes to hysterectomy including rarely identified longerterm outcomes such as later pelvic floor dysfunction or prolapse. We did not however include sexual function, quality of life outcomes, or time to return to full function/work.

We recognize that despite this study being one of the largest single practitioner studies comparing vaginal hysterectomy outcomes, unlike multi-practitioner studies, our comparison of morbidity between subgroups demonstrates a rarity of complications making it difficult to identify true differences that this study, less powered, might otherwise have clarified. Nevertheless, this study, representing a single practitioner, community-based data set, appropriately represents the conditions, circumstances, and potential outcomes for many gynecologic surgeons facing the challenge of increasing their vaginal hysterectomy rate.

We do not address the potentially doubly confounding influence on outcomes represented by either the presence of prolapse or the corrective procedures often performed for it in addition to hysterectomy. Cases where prolapse is the indication for hysterectomy may benefit in terms of outcome due to the associated technical ease of access to the uterus and cervix. More common in the standard group, prolapse could also result however in higher morbidity where adjunct resuspension procedures are necessarily performed

In our analysis, comparing non-standard hysterectomy cases requiring conversion to those without conversion, neither fibroid enlargement, oopherectomy or prior cesarean were clearly predictive of conversion, nor did conversion to abdominal route of itself result in significant morbid sequelae; for the 13 Non-standard cases which necessitated conversion to abdominal route, prolonged OR time, an increased EBL without transfusion and a slightly prolonged LOS were the only significant differences. Indeed in the properly counseled patient, conversion need not represent a regrettable outcome. Going forward, we can also see the increased role of laparoscopy in those rare situations in which conversion might become necessary - a "laparoscopic assist" only, as some authors suggest, when necessary and not as precondition for a vaginal approach [22]. We do understand that statistical analysis within the subset of cases converted to abdominal route lacks the power to make particularly strong conclusions.

This study is not of prospective design. Arguably, the retrospective comparison between groups is simply one of convenience and of no import. Not formally prospective, this study nevertheless represents a retrospective analysis of a prospective intent to perform with few exceptions all cases vaginally and thus approaches the rigor of a truly prospective study. Our comparison therefore is not one of convenience but one that, as credible as a prospective analysis might be, indicates that performing vaginal hysterectomy in patients with one or more of prior cesarean, enlarged uteri $>14$ weeks, or need for oophorectomy while possibly fraught with increased difficulty is nevertheless not fraught with more complications or greater real morbidity.

\section{Conclusion}

This study identifies the relative safety and acceptability of an inclusive strategy for selection of vaginal route hysterectomy and challenges the three most common contraindications to vaginal hysterectomy. By such a strategy, we have achieved a rate of vaginal hysterectomy beyond $95 \%$ with the only clinically significant complications being a very low rate of conversion to abdominal route that, without greater morbid sequelae, is preferable to primarily choosing an abdominal or laparoscopic route for all.

Acknowledgments This work was done under the auspices of Dartmouth Hitchcock/Cheshire Medical Center, Keene, NH, USA.

Disclosure of interests No conflict of interest.

Contribution to authorship Substantial writing, editing, literature review, and data analysis were contributed by Dr. Johnson. Study concept, clinical work, data extraction, data entry, statistical analysis, and majority writing and editing were contributed by Dr. Mackenzie.

Details of ethics approval The approval was through the Committee for the Protection of Human Subjects, Dartmouth Hitchcock Medical Center January 2009. 
Funding There was no funding.

Declaration of interest The authors report no conflicts of interest. The authors alone are responsible for the content and writing of the paper.

\section{References}

1. Wu JM, Wechter ME, Geller EJ, Nguyen TV, Visco AG (2007) Hysterectomy rates in the United States, 2003. Obstet Gynecol 110:1091-1095

2. Whiteman MK, Hillis SD, Jamieson DJ, Morrow B, Podgornik MN, Brett KM et al (2008) Inpatient hysterectomy surveillance in the United States, 2000-2004. Am J Obstet Gynecol 198:34.3e1-34e7

3. Garry R, Fountain J, Mason S, Hawe J, Napp V, Abbott J et al (2004) The eVALuate study: two parallel randomized trials, on comparing laparoscopic with abdominal hysterectomy, the other comparing laparoscopic with vaginal hysterectomy. BMJ 328:129-138

4. Lumsden MA, Twaddle S, Hawthorn R, Traynor I, Gilmore D, Davis $\mathrm{J}$ et al (2000) A randomised comparison and economic evaluation of laparoscopic-assisted hysterectomy and abdominal hysterectomy. BJOG 53:214-219

5. Reich H, Roberts L (2003) Laparoscopic hysterectomy in current gynaecological practice. Rev Gynaecol Pract 3:32-40

6. Altgassen C, Michels W, Schneider A (2004) Learning laparoscopic assisted hysterectomy. Obstet Gynecol 104:308-313

7. Kovac SR (2000) Hysterectomy outcomes in patients with similar indications. Obstet Gynecol 95:787-793

8. Van Den Eeden SK, Glasser M, Mathias SD, Colwell HH, Pasta DJ, Kunz K (1998) Quality of life, health care utilization, and costs among women undergoing hysterectomy in a managed-care setting. Am J Obstet Gynecol 178:91-100

9. Mulholland C, Harding N, Bradley S, Stevenson M (1996) Regional variations in the utilization rate of vaginal and abdominal hysterectomies in the United Kingdom. J Public Health Med 18:400-405

10. Choosing the route of hysterectomy for benign disease (2009) ACOG Committee Opinion No. 444. Obstet Gynecol 114 (5):1156-1158

11. Kovac SR (1995) Guidelines to determine the route of hysterectomy. Obstet Gynecol 85:18-23

12. Varma R, Tahseen S, Lokugamage AU, Kunde D (2001) Vaginal route as the norm when planning hysterectomy for benign conditions: change in practice. Obstet Gynecol 97:613-616

13. Davies A, Vizza E, Bournas N, O’Connor H, Magos A (1998) How to increase the proportion of hysterectomies performed vaginally. Am J Obstet Gynecol 179:1008-1012

14. Magos A, Bournas N, Sinha R, Richardson RE, O'Connor H (1996) Vaginal hysterectomy for the large uterus. British J Obstet Gynecol 103:246-251

15. Hoffman MS (1991) Transvaginal removal of ovaries with endoloop sutures at the time of transvaginal hysterectomy. Am J Obstet Gynecol 165:407-408

16. Kovak SR, Cruikshank SH (1996) Guidelines to determine the route of oopherectomy with hysterectomy. Am J Obstet Gynecol $175: 1483-1488$

17. Hoffman MS, Jaeger M (1990) A new method for gaining entry into the scarred anterior cul-de-sac during transvaginal surgery. Am J Obset Gynecol 162:1269-1270

18. Doucette RC, Sharp HT, Alder SC (2001) Challenging generally accepted contraindications to vaginal hysterectomy. Am J Obstet Gynecol 184:1386-1391

19. Tu FF, Beaumont JL, Senapati S, Gordon TEJ (2009) Route of hysterectomy influence and teaching hospital status. Obstet Gynecol 114:73-78

20. Dunn TS, Weaver A, Wolf D, Goodard W (2006) Vaginal hysterectomies performed in a residency program: can we increase the number? J Reprod Med 51:83-86

21. Kovac SR (1999) Determining the route and method of hysterectomy. Key Clinical Decision Series-Ethicon EndoSurgery ISBN 0-9673302-0-3

22. Richardson RE, Bournas N, Magos AL (1995) Is laparoscopic hysterectomy a waste of time? Lancet 345:36-41 